\title{
ASTEROIDS
}

\section{Cometary Bennu?}

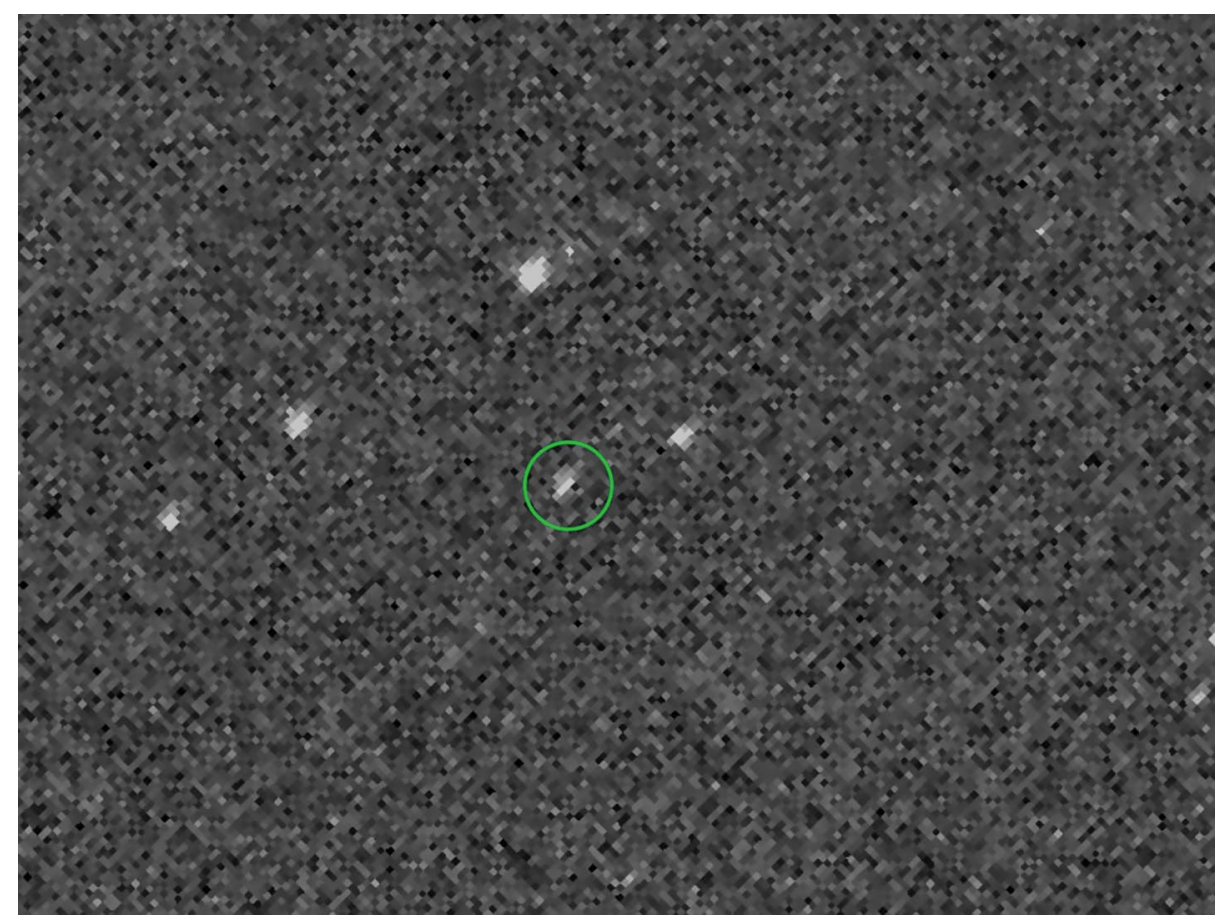

Credit: NASA/Goddard/University of Arizona

Asteroid (101955) Bennu is the target of NASA's sample-return mission OSIRIS-REx (the first image of the asteroid obtained by the spacecraft is pictured). In anticipation of the rendezvous, which will happen in December this year, many Earth-based observations of the body have been performed. Such measurements will help place the upcoming in situ measurement within the wider context of remote monitoring of asteroids from Earth. Alberto Cellino and collaborators find some unexpected behaviour by looking at Bennu's polarimetry.

Phase-polarization curves of asteroids, which trace their polarization as a function of the Sun-asteroid-Earth angle (the phase angle), help determine their surface properties, such as the albedo or the grain size of their regolith layer. Cellino et al. analyse seven polarization measurements of Bennu obtained by the FORS2 instrument at the Very Large Telescope (VLT) between
February and June 2018, covering phase angles between $\sim 15^{\circ}$ and $\sim 60^{\circ}$. Among the asteroids with the same spectral behaviour as Bennu - known as B-types, corresponding to primitive carbonaceous chondrites - only 3200 Phaethon has been observed at such a wide range of phase angles.

The results show that Bennu's phase curve is more similar to that of comet Hale-Bopp than of Phaethon. Thus, Bennu might belong to a sub-class of near-Earth objects with cometary characteristics or of cometary origin. The close-up observations of OSIRIS-REx will determine if Bennu is actually a member of the elusive category of objects between standard asteroids and standard comets.

\section{Luca Maltagliati}

Published online: 24 September 2018 https://doi.org/10.1038/s41550-018-0599-5 